\title{
Dynamic Hot Spot Prediction by Learning Spatial-Temporal Utilization of Taxiway Intersections
}

\author{
Hasnain Ali*, Raphael Delair ${ }^{\dagger}$, Duc-Thinh Pham*, Sameer Alam*, Michael Schultz \\ *Nanyang Technological University \\ \{hasnain@e.,dtpham@,sameeralam@\}ntu.edu.sg \\ $\dagger$ Ecole Nationale de l'Aviation Civile (ENAC), French Civil Aviation University \\ \{delair.raphael\}@gmail.com \\ $\ddagger$ Institute of Logistics and Aviation, Dresden University of Technology \\ \{michael.schultz\}@tu-dresden.de
}

\begin{abstract}
Airports across the world are expanding by building multiple ground control towers and resorting to complex taxiway and runway system, in response to growing air traffic. Current outcome- based ground safety management at the airside may impede our potential to learn from and adapt to evolving air traffic scenarios, owing to the sparsity of accidents when compared with number of daily airside operations. To augment airside ground safety at Singapore Changi airport, in this study, we predict dynamic hot spots- areas where multiple aircraft may come in close vicinity on taxiways, as pre-cursor events to airside conflicts. We use airside infrastructure and A-SMGCS operations data of Changi airport to model aircraft arrival at different taxiway intersections both in temporal and spatial dimensions. The statistically learnt spatial-temporal model is then used to compute conflict probability at identified intersections, in order to evaluate conflict coefficients or hotness values of hot spots. These hot spots are then visually displayed on the aerodrome diagram for heightened attention of ground ATCOs. In the Subjective opinion of Ground Movement Air Traffic Controller, highlighted Hot Spots make sense and leads to better understanding of taxiway movements and increased situational awareness. Future research shall incorporate detailed human-in-the-loop validation of the dynamic hot spot model by ATCOs in 360 degree tower simulator.
\end{abstract}

Index Terms-Airside Operations; Taxiway; Hot spot; Singapore Changi Airport

\section{INTRODUCTION}

International Civil Aviation Organisation (ICAO) monitors aviation safety under its' Global Aviation Safety Plan (GASP) [1]. After analysing safety incidents that have occurred in the current decade, ICAO has identified three high-risk accident occurrence categories viz. Runway Safety (RS) related events, Loss of Control In-Flight (LOC-I) and Controlled flight into terrain (CFIT). In its' latest report (in year 2019), ICAO has published that RS related events, which includes ground collision and collision with obstacles, accounted for nearly half of all accidents in 2017 and 2018. In fact, RS related events lead to the highest percentage of the accidents which destroyed or caused substantial damage to aircraft (see Fig. 1). With air traffic levels expected to double in next two decades [2], airports across the world will witness upsurge in airside ground movements as a direct result of increased arrivals and departures. Moreover, to cater to the growing traffic, airports are expanding and augmenting their air traffic control capabilities by building multiple airport control towers and resorting to complex taxiway and runway system designs. Aviation authorities have expressed their predicaments about airports' and air traffic control's current incapability to cope up with the growing demand [2]; and if not prepared for, well in advance, this may clearly lead to much higher RS related incidents. The unprecedented traffic growth coupled with complex airside design shall pose serious threats to the safety of day-to-day ground operations. Thus, there is a growing and urgent need of research in this area to equip Airport traffic controllers (ATCOs) and pilots for the future safety challenges. In other words, research efforts that can assist ATCOs and pilots in identifying safety challenges, before accidents even may occur, will be vital for efficient and safe airport airside operations.

Although, ICAO has been analysing and publishing safety reports every year (since 2011), its' appraisal of global and regional aviation safety is based on outcome (incidents or accidents) metrics, that draws inspiration from long-established view on safety as absence of loss or unsafe incidents. As such, owing to the sparsity of accidents when compared with number of daily airside operations, this current safety management approach may impede our potential to learn from daily operations, moving ahead into the future. Aviation safety management therefore, must shift from a compliancebased approach to a performance-based approach [3]. In other words, the reactive traditional view of safety, as absence of accidents (safety compliant), needs to transition towards a proactive monitoring of indicators [4], [5] of safety performance (performance-based). This will help to proactively identify and prevent errors in the quickly evolving traffic scenarios on airports.

The biggest enabler to the proactive incident identification, would be our ability to learn from daily airside ground surveillance data and tools that assist ground safety monitoring. 

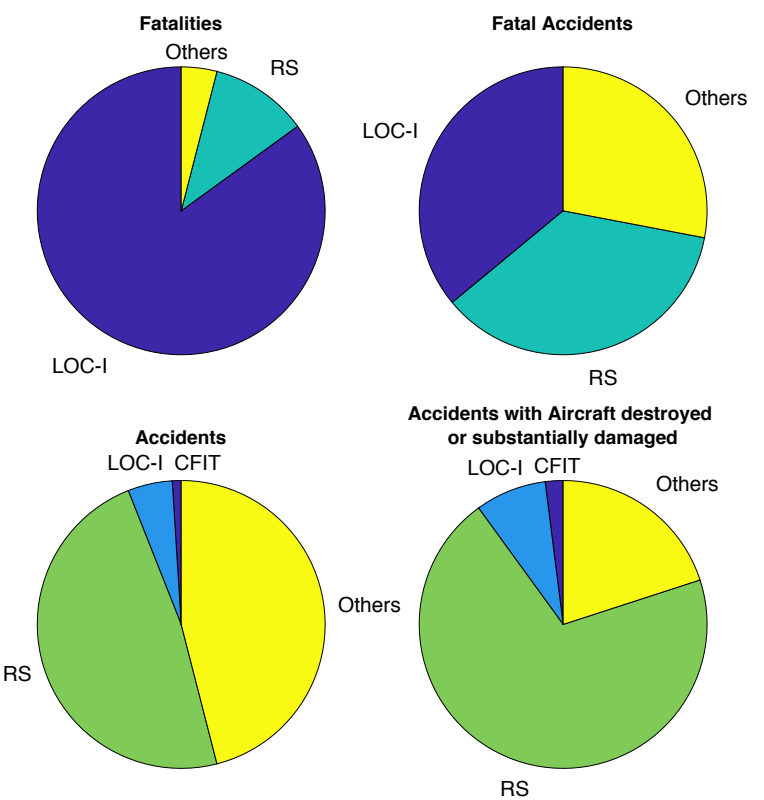

Fig. 1. Contribution of RS related events (including collision with obstacles and ground collisions) to fatalities and aircraft damage. RS: Runway Safety related events, LOC-I: Loss of Control In-Flight and CFIT: Controlled flight into terrain. Source: ICAO global aviation safety report, 2019.

Advanced Surface Movement Guidance and Control System (A-SMGCS), is one such tool that, provides surveillance on the runway, taxiways, stands etc. to ATCOs. A-SMGCS data contains second by second update of all ground movements captured by a full suite of cooperative (like Multilateration, ADS-B, Vehicle Tracking systems, Secondary Surveillance Radar) \& non-cooperative (like Surface Movement Radar, Primary Surveillance Radar) sensors. These sensors capture information about ground vehicles and aircraft on the maneuvering area, like aircraft/vehicle identification, flight plan, type of aircraft, location, and time stamps. This information has been used, in the past research [6], to model aircraft's spatial temporal movement on airport-airside from the assigned source to destination. This model can now be leveraged to statistically predict the concurrent presence of multiple aircraft in the close vicinity- hot spots, which may be closely monitored as precursor events by ATCOs to prevent ground collisions and conflicts.

\section{Literature REvieW}

\section{A. Hot Spots}

ICAO defines a hot spot to be a "location on an airport movement area with a history of potential risk of collision or runway incursion, and where heightened attention by pilots and drivers is necessary" [7]. Conventionally, a hot spot (refer Fig. 2) is a complex or confusing taxiway-taxiway or taxiwayrunway intersection where surface incidents in past have taken place. These incidents typically occur as a result of problems in airport layout, traffic flow, airport markings, situational awareness etc. Identification and subsequent publication of airport hot spots, as evidence suggests, raises runway threat awareness [8]. Displaying hot spot information on airport diagram, alerts airport users of any unsafe areas and assists them in planning ground movements. By proper planning of ground movements, pilots and ATCOs add safety nets to overall airport operations.

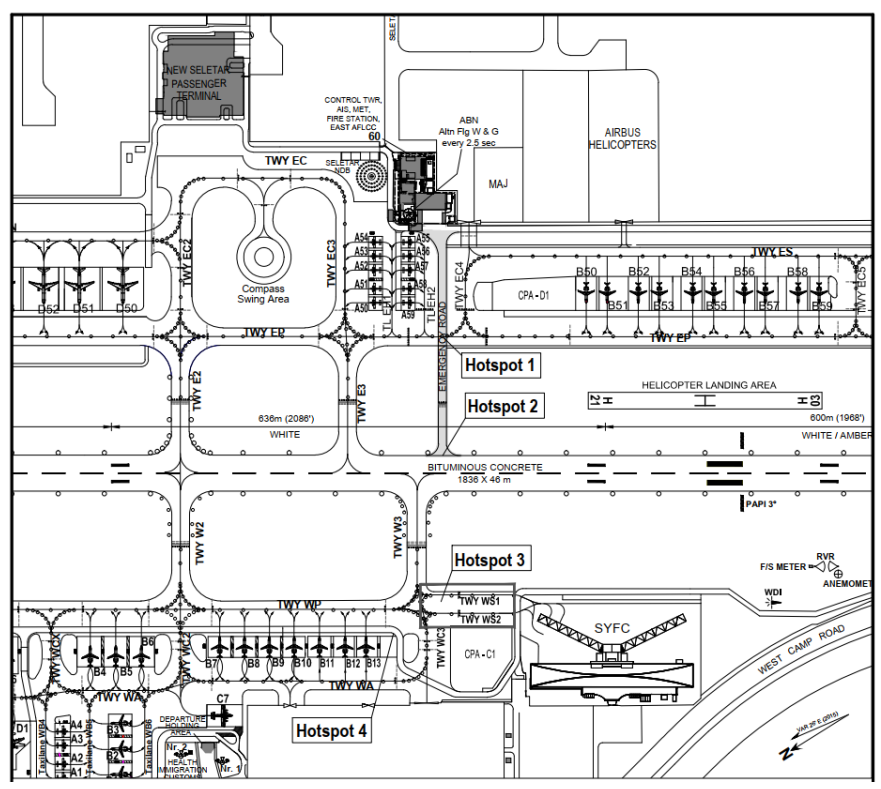

Fig. 2. Hot spots at Singapore Seletar Aerodrome. Static hot spots, owing to their generic nature, are of limited use for aircraft not maneuvering through them.

Fig. 2 represents four regions identified as hot spots at Singapore Seletar Aerodrome. Aerodrome users are cautioned to manoeuvre through these regions with due care and extra precautions. However, these static hot spots are of limited use for aircraft not maneuvering through them. Moreover, these regions become prone to collisions only under certain circumstances which are detailed in the explanatory notes accompanying the hot spot chart.

\section{B. Runway Incursions}

Owing to high energy (landing/take-off) aircraft interactions at runways, incursions have garnered a lot of academic interest. In particular, runway incursions are "occurrences at an aerodrome involving the incorrect presence of an aircraft, vehicle or person on the protected area of a surface designated for the landing and take off of aircraft" [7]. Runway incursions are precursor events or causal factors of runway collisions. The underlying assumption in studying incursions, is that by reducing the number of runway incursions, airport users can bring down runway collisions, as well. Although pilot deviations, and/or vehicle/pedestrian deviations cause majority of the incursions, FAA statistics reveals that the operational errors (related to ATCOs) and pilot deviations make up the major part of the serious runway incursions of types A and B [9]. Researchers in [10] performed statistical analysis to determine relationship between airport geometry factors and the number of runway incursions. They found 
SPATIAL
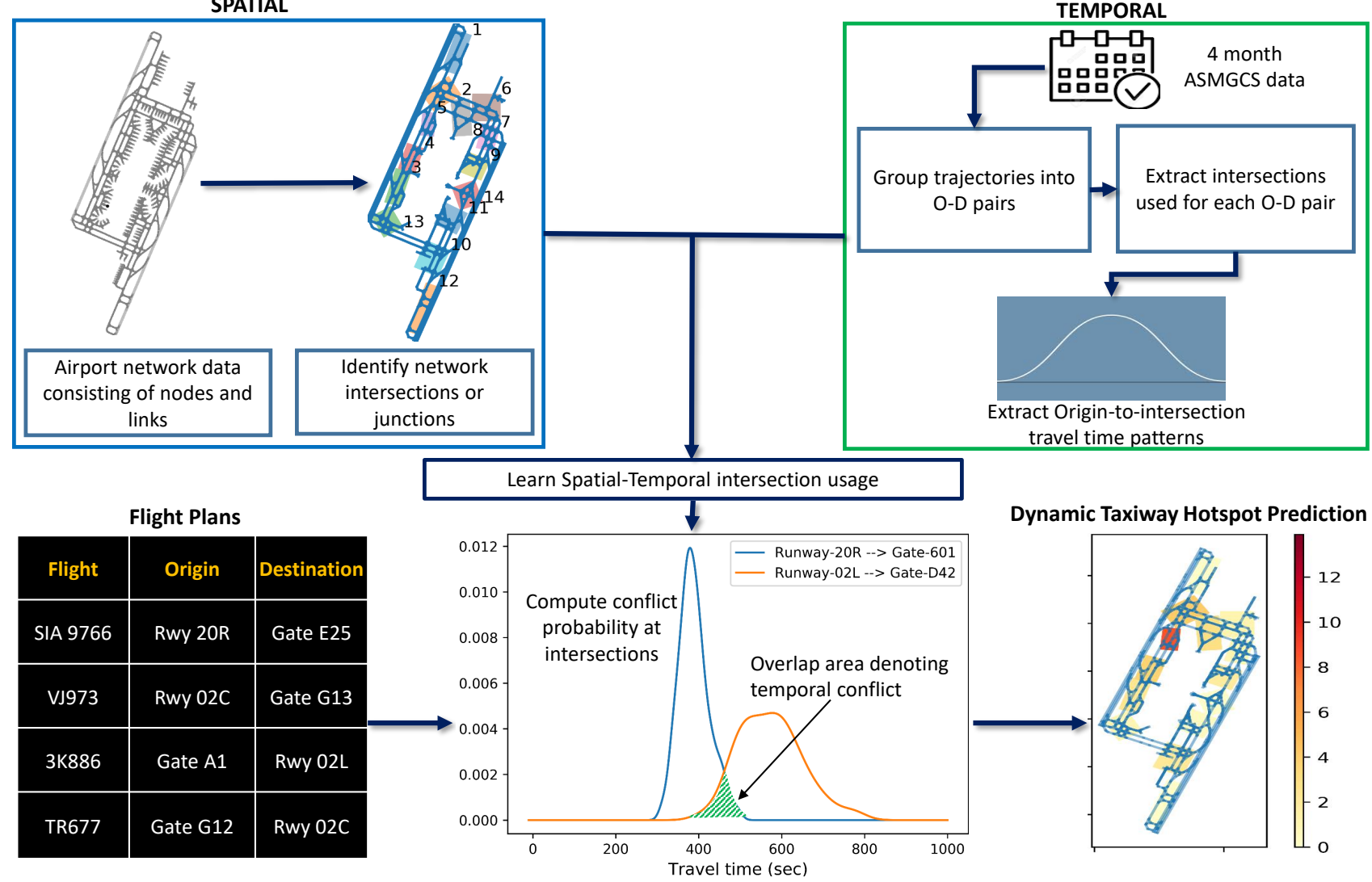

Fig. 3. Concept diagram for dynamic hot spot prediction model, illustrating interactions between spatial (blue box) and temporal (green box) sub-components which are employed on a day's scheduled operations to obtain dynamic hot spots on taxiways.

intersecting runways, number of crossing taxiways per runway and the number of runway intersections per runway to be significantly impacting incursion rates. Drifting further from the standard outcome based analysis of safety events on the runway, researchers in [11] assessed runway incursions using agent-based dynamic risk modelling to determine collision probability.

Incursion avoidance research fundamentally observes two kinds of approaches- stopping an aircraft from entering an active runway; and detecting an imminent runway incursion as early as possible [9]. Current methods employ safety alerts against operational errors such as raising alarms when Air Traffic Controllers (ATCOs) commission more than one aircraft to use an active runway. Humans (expert ATCOs) play an important role in current runway incursion prevention systems and it is found that situational awareness is key to prevent incursions. Final Approach Runway Occupancy Signal (FAROS) and Runway Status Lights (RWSL), for instance, have been found to reduce runway incursions by $70 \%$ at the Dallas/Fort Worth International Airport. Preventing runway incursions, therefore largely depend on the surveillance system that provides input to the systems and/or ATCs dealing with this problem. Much of the effort has thus gone into development of a reliable, effective and accurate surveillance system with a coverage of the maneuvering area of an airport [12][15].

\section{Taxiway Incurions}

Taxiway surveillance data, in the past studies, has been used to model stochastic airside operations. Researchers in [16], modelled aircraft manoeuvre on taxiways, using Airport Surface Detection Equipment, Model X (ASDE-X) data. They modelled taxiway travel time based on probability distributions i.e. unimpeded taxiway travel time using Erlang distribution; number of stops as geometric random variable; and stationary time corresponding to these stops as exponential random variable; after observing ground movements. Using the model, the authors could maintain steady traffic level with significant reduction in aircraft surface fuel burn. Airport surveillance radar data has also been used to assess ground movements for safety [17], [18] purposes. For instance, [18] quantitatively modelled collision likelihood between aircraft using twelve week ASDE-X data of 35 US airports. As a proxy for collisions, the study identified potentially hazardous interactions (PHIs) between taxiing aircraft where the separation and convergence dynamics indicate a non-negligible probability of collision. The results showed that congestion near runway hold lines and at transitions between taxiways and ramps were the most commonly observed symptoms of increased 


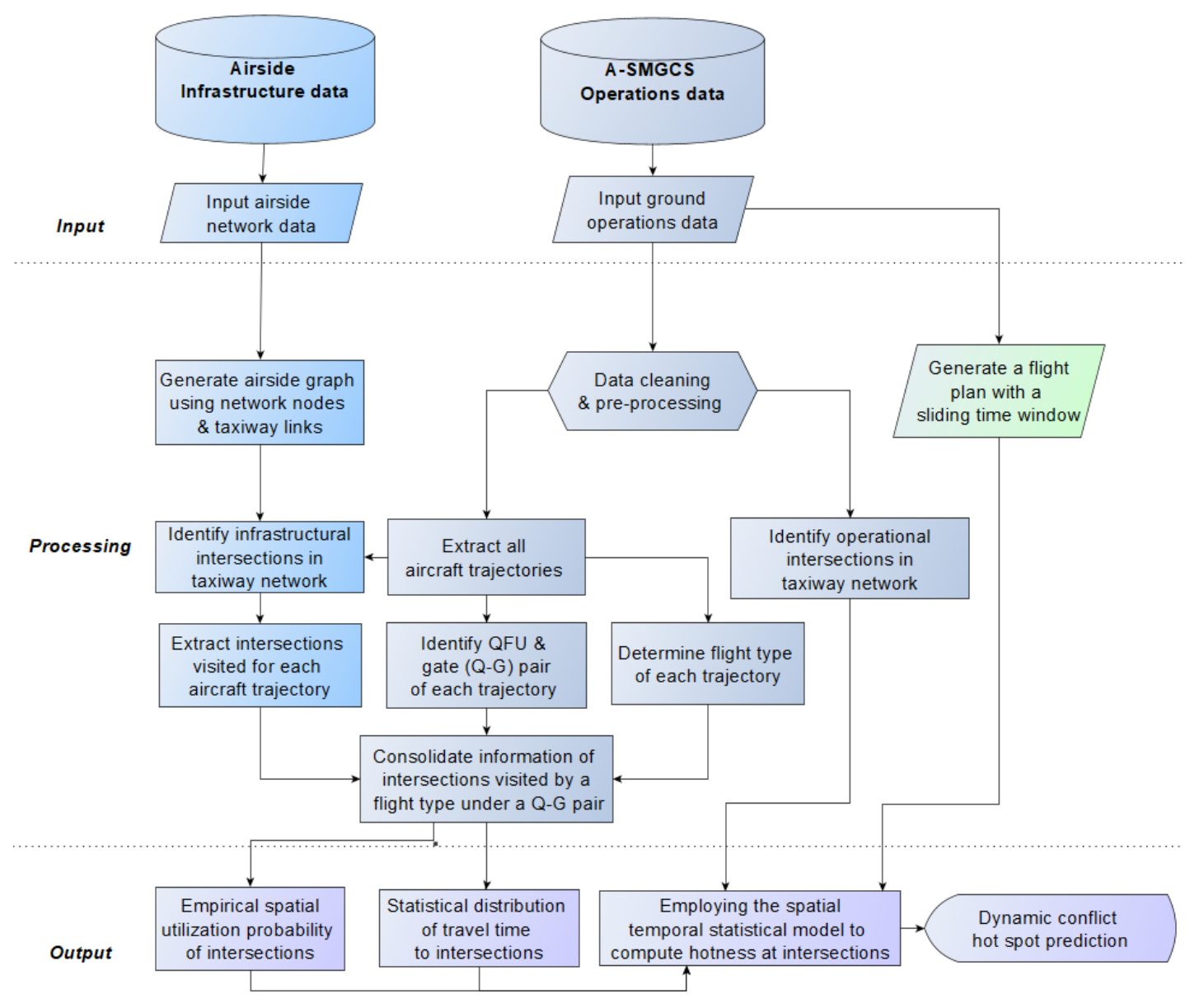

Fig. 4. Methodology to predict hot spots using airside infrastructure and A-SMGCS operations data.

collision potential. Recently, [19] used aerodrome surveillance radar (ASR) data to identify conflicts. Conflicts, in their model, arose when safe separation and heading threshold was violated between 2 aircraft, using the same taxiway. Clearly most of these studies, are performed with objectives like reducing delays [20] [21], emissions etc. which do not have safety as their prime concern. Consequently, the suggested operations, do not adress safety concerns. Other the other hand in the past research studies, where authors have focused on safety issues, these studies either are retrospective in naturewhere problematic interactions are identified in the past, or are formulated around a rule based model which does not include the stochastic nature of ground operations. Ground surveillance data, however, can be better leveraged to predict hot spots which changes in time and space dynamically, based on ground operations changing with time.

In this study, we extend ICAO's definition of hot spot to include areas on the Changi aerodrome taxiway, where multiple aircraft come in close vicinity as this situation might potentially lead to conflicts, if not resolved by ATCOs. Unlike the currently defined hot spots at airports, which are generic and static for all aircraft present at the airport, hot spots in this study are dynamic as these appear and fade in time and space based on aircraft trajectory interactions, and therefore, must attract heightened attention by pilots and ATCOs. Moreover, for the purpose of this study, we use the term 'predict' hot spots hereafter, to specifically mean identifying hot spots in a given look-ahead time. These hot spots are highlighted based on conflict coefficient or hotness values of intersections which is evaluated probabilistically based on planned aircraft movements between runways and gates.

\section{PRoBlem Formulation}

In Fig. 3, we illustrate the proposed framework for predicting dynamic hot spots at airside taxiways. The framework consists of spatial and temporal sub-components, which represent the spatial-temporal movements of aircraft on the ground. Blue box in the upper left corner illustrate the spatial sub-component. We take the airside structure and model it as a network consisting of links and nodes. After analysing the network for nodes connecting multiple links, we identify intersections or junctions on airport taxiways, where ground traffic merges. These intersections are the potential hot spots.

On the other hand, green box in the upper right corner represents the temporal sub-component. We first categorize all the observed trajectories based on the origin and destination 
(runways and gates in airside context) pairs they share. Then, for all the aircraft corresponding to each trajectory category, we identify the possible subset of intersections visited in the past. We then compute the travel time distribution to each intersection for all the trajectory categories, from their respective origin points. In other words, aircraft journeys to all the potential hot spots are statistically learnt in time.

Using both the components learnt above, we preserve the knowledge of intersection usage using probabilistic kernel density estimates. After learning the spatial temporal information, which interact to give rise to the hot spots, the learnt spatial temporal model is employed on a day's scheduled ground operation plan. Based on a sliding window algorithm, we compute the probability of concurrent presence of multiple aircraft at an intersection in a given time frame. This is translated mathematically into a conflict coefficient or hotness value for each intersection that represents how busy or hot an intersection will become through the course of planned operations. This is done for all flights in a time window to generate a heat map that dynamically updates as ground traffic merges and re-organises on the taxiway system through the day.

\section{Methodology}

The detailed step-by-step procedure to predict dynamic hot spots at (Changi) airside is described in this section. Refer Fig. 4 to view the methodological flowchart. The input to the model are infrastructural and operations data- these are discussed in IV-A. Next, in the processing stage (refer section IV-B to IV-I), input data are used to get the spatial and temporal intersection usage, probable aircraft conflicts and this finally leads to the prediction of dynamic hot spots as output (refer section IV-J to IV-M) for scheduled ground operations.

\section{A. Data}

Following data is input to the model used to compute dynamic hot spots.

1) Airside Infrastructure: An airport airside modelled as a network, is composed of nodes which are connected through links. Node description- in the form of co-ordinates \& link information- in the form of arcs joining nodes and direction of these arcs (clockwise or counter-clockwise) is used to generate network graph later.

2) A-SMGCS Operations: In this study, 4 month (Oct2017 to Jan-2018; 121 days) A-SMGCS data, detailing Changi airside operations, has been used.

Directly available information:

1) Date

2) Flight call sign and type of aircraft

3) Time stamp (captured data is updated every second)

4) Location Stamp (Latitude and Longitude)

5) Assigned gate or stand

6) Mean flight level or altitude

Inferred information:

1) Whether the aircraft serves arrival or departure flight inferred from Source/Destination airport

\section{B. Data Cleaning and Pre-processing}

As the aim of the study is to predict hot spots on taxiways, following data was filtered out:

1) En-route/In-the-air trajectories: Data points above the airport elevation level, were excluded.

2) Ground vehicle trajectories: Unlike aircraft, vehicle trajectory itinerary (source-destination) is not mentioned in flight plan. To simplify the analyses therefore, ground vehicles are excluded from the analyses.

3) Incomplete ground trajectories: Not every trajectory information is complete. After initial observations, aircraft trajectories which have less points (fewer than 10) are excluded from further analysis. This, also ensures ease of determining magnetic orientation of runway (QFU; refer IV-G) later.

The 121 days' pre-processed ground data contains 62.6 million rows, detailing the location and time stamps of aircraft trajectories along with flight schedule information. This information corresponds to more than 110,000 unique aircraft trajectories. On an average, 900-1000 flights land at Changi airport every day.

\section{Generate Airside Graph}

Airside infrastructure data (refer IV-A1) is used to generate airside graph. This graph (refer Fig. 5) is further employed to analyze aircraft operational trajectories on the fixed airside layout. In other words, the underlying graph structure helps to explain the interactions between airport network and aircraft operations, by describing the physical constraints to airside movements.

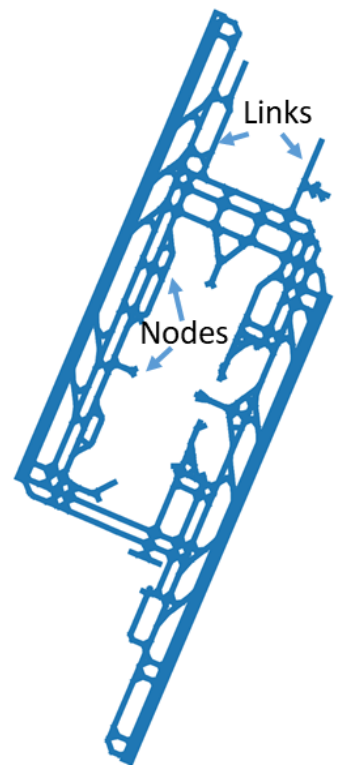

Fig. 5. Changi airside structure which, modelled as a network, is composed of nodes which are connected through taxiway and runway links.

\section{Identify Infrastructural Intersections}

Any (airside) network contains intersection (or junction) nodes which may be accessed from multiple directions by 
aircraft maneuvering in the airside. These nodes represent crossing taxiways. Fig. 6 represents all the 394 nodes present at Changi airside taxiway. To identify intersection nodes, taxiway network nodes that have 3 (T-junction) or 4 degrees (4-way junction) are identified. Degrees refer to the number of connecting links.

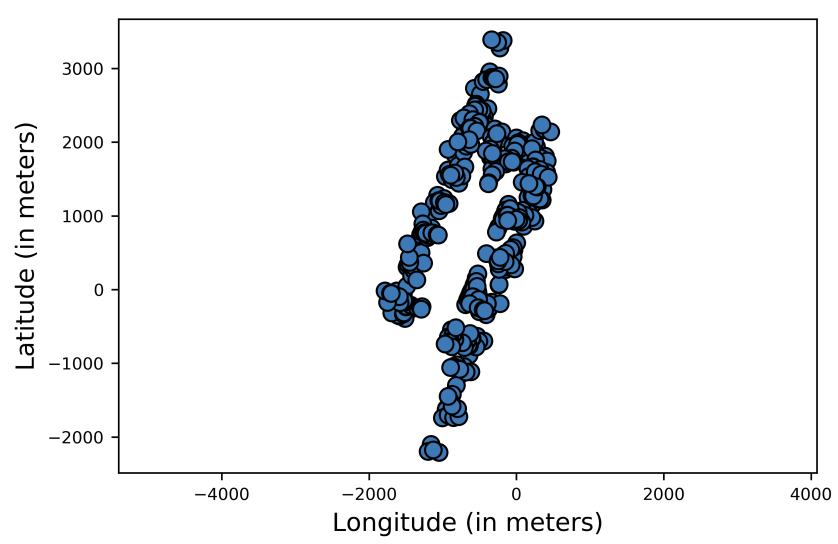

Fig. 6. Intersection nodes existing at Changi taxiway network

An (airside) intersection, in this study for monitoring purposes, is defined to be an area consisting of multiple intersection nodes. Since clustering algorithms are generally heuristic in nature [22] and are often polynomial in time, Agglomerative clustering [23] is applied to group the intersection nodes into intersections (bigger areas), which are potential hot spots. These intersections, which may experience conflicts when visited concurrently by multiple aircraft, are the points of interest. Fig. 7 represents 14 intersections identified, for monitoring purposes, at Changi airport taxiway network.

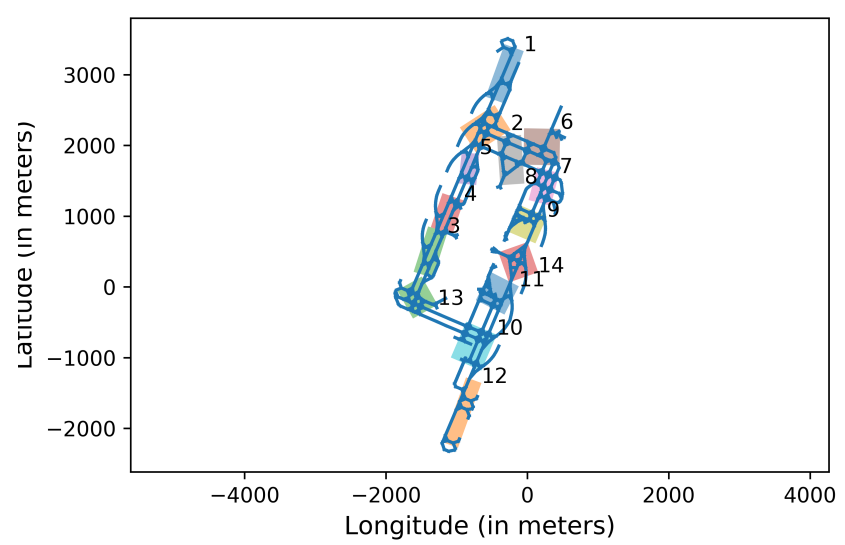

Fig. 7. Intersections identified at Changi taxiway network: potential hot spots. Each colored box represent an intersection.

\section{E. Extract Aircraft Trajectories}

The pre-processed A-SMGCS operations data contains multiple aircraft movements (or trajectories) with many aircraft, serving both arrival and departure flights, and frequenting the airport on multiple days, over a period of 4 months. The data is therefore decomposed into unique aircraft trajectorieseach trajectory representing an aircraft serving an arrival or departure flight, at a time, in Changi airport.

\section{F. Determine Flight Type}

Each aircraft- represented by a trajectory- either serves an arrival or departure flight. Therefore flight type information refers to ascertaining if a flight is an arrival flight or a departure flight. This information can be inferred from operations data (refer IV-A2).

\section{G. Identify $Q F U$ and Gate $(Q-G)$ Pair}

On airside, an arrival trajectory originates from runway and terminates in gate at apron. On the other hand, a departure trajectory originates from gate at apron and terminates in runway. Thus, every trajectory can be categorized by its QFUGate (Q-G) pair. Gate information is given in A-SMGCS data (refer IV-A). However, runway-in-use (QFU) information is not available directly in the data. Therefore QFU is derived from the initial (for arrivals) or final (for departures) location stamps of aircraft trajectory, following the below procedure.

QFU (magnetic orientation of runway) essentially refers to 2 things- runway and it's magnetic orientation in active usage. To determine the runway used, proximity of the trajectory location stamp points to each runway is checked. The closest runway is assigned to the trajectory. Further, as runway configuration changes, during the day, may alter the ground journeys between the same runway and gate, another parameter, QFU should be learnt for each aircraft by observing direction progression from the first few (10) data points for arrival flights and the last few (10) data points for departure flights. Although 2 points would have sufficed to compute aircraft movement direction vector, the lower limit of 10 is set to account for any noise in the data set.

\section{H. Extract Intersections Visited}

Each aircraft visits a subset of 14 intersections (identified in subsection IV-D), during its' movement between gate and QFU. For each historical aircraft trajectory, intersections visited are observed in operations and this information is saved.

\section{Intersection Shared by a $Q-G$ pair}

Each aircraft, now characterized by a unique Q-G pair, may only visit a subset of all available intersections. Moreover, a variety of route options exist between a Q-G pair, and depending on the opted route, intersections visited may vary even for a unique $\mathrm{Q}-\mathrm{G}$ pair. Moreover, depending on flight type (arrival or departure), order in which intersections are visited between a Q-G pair will differ and this impacts the travel time to the intersections. Thus, the set of all intersections that have been visited for a $\mathrm{Q}-\mathrm{G}$ pair by a flight type, is extracted by observing past flight trajectories in the 4 month operations (A-SMGCS) data. 


\section{J. Empirical Utilization (spatial) of intersections}

It can be observed that for every QFU-Gate-Flight type combination (Q-G-F) identified in last sub-section (IV-I), not all intersections corresponding to a Q-G-F are frequented equally (i.e. with equal probability). This implies that some routes between a Q-G pair are preferred over others. Thus, given a Q-G-F, utilization probability of an intersection- $P_{i}$ is computed using equation 1.

$$
P_{i}=\frac{n_{i}}{\sum_{i \in I_{Q G F}} n_{i}}
$$

Where,

- $n_{i}$ represents number of times intersection $i$ is visited.

- $I_{Q G F}$ represents set of all intersections observed for a Q-G-F.

\section{K. Distribution of Travel Time to Intersections}

Based on past journeys, time taken by a Q-G-F combination to each intersection can be statistically learnt. This statistical information can then be stored by means of fitting kernel density estimate (KDE) to all the empirical travel time observations [24], [25]. This shall be used for computing conflict probability at an intersection later. Fig. 8 shows KDE fitted to empirical observations of travel time to intersection 5 (representative) between different Q-G pairs.

\section{Import Flight Schedule with a Sliding Time Window}

To compute hotness arising due to aircraft intersections at the taxiways, we import a flight schedule and run a sliding time window algorithm to obtain a set of departure and arrival flights as input to the dynamic hot spot model. The sliding window is 10 minutes long which includes the past 30 minute traffic history to compute intersection hotness. The window shifts by 30 minutes in time after one computation cycle. In other words, starting at $10 \mathrm{AM}$, the sliding window shall predict hot spots until 10:10 AM, considering traffic that is active since 9:30 AM and slides to 10:30 $\mathrm{AM}$ in the next computation cycle.

\section{Predict Dynamic Hot Spots}

1) Spatial conflict: For a conflict to occur, two (or more) aircraft must come at an intersection together. Spatial conflict $\left(C_{s, i}\right)$ between two flights $a$ and $b$, at an intersection $i$, then can be computed as follows.

$$
C_{s, i}=P_{i}(\alpha \cap \beta)
$$

Where,

- $\alpha$ is the event when flight $a$ arrives at intersection $i$.

- $\beta$ is the event when flight $b$ arrives at intersection $i$.

- $i$ is a common intersection between Q-G-F that flights $a$ and $b$ correspond to respectively.

Further, it is reasonable to assume that events $\alpha$ and $\beta$ are conditionally independent, i.e. flight routes are independent and in absence of any explicit hold orders from ATCOs, flights may arrive at an intersection at their usual times (statistical observations). Therefore equation 2 , can be simplified as follows

$$
C_{s, i}=P_{i}(\alpha) * P_{i}(\beta)
$$

Spatial conflict, in of itself, is not sufficient for a collision to occur. If flights arrive at an intersection at different time points, then no collision may take place. Hence, for a ground collision, flights must also arrive at the same intersection concurrently. Thus, in the following text, we compute temporal conflict.

2) Temporal conflict: The learnt statistical KDEs (in subsection IV-K) can be used to compute temporal conflict probability- the overlapping area between KDEs (representing active Q-G-F) drawn at their respective start times in a given time frame. Temporal conflict $\left(C_{t}\right)$ between two flights $a$ and $b$, at an intersection $i$, then can be computed as follows.

$$
C_{t, i}=\varphi_{i}(a, b)=\int_{t_{s}}^{t_{e}} \min (f(t), g(t)) d t
$$

Where,

- $\varphi_{i}(a, b)$ refers to common area between KDEs corresponding to flights $a$ and $b$.

- $f(t)$ represents KDE corresponding to flight a.

- $g(t)$ represents KDE corresponding to flights $\mathrm{b}$.

- $t_{s}$ represents start time of overlap.

- $t_{e}$ represents end time of overlap.

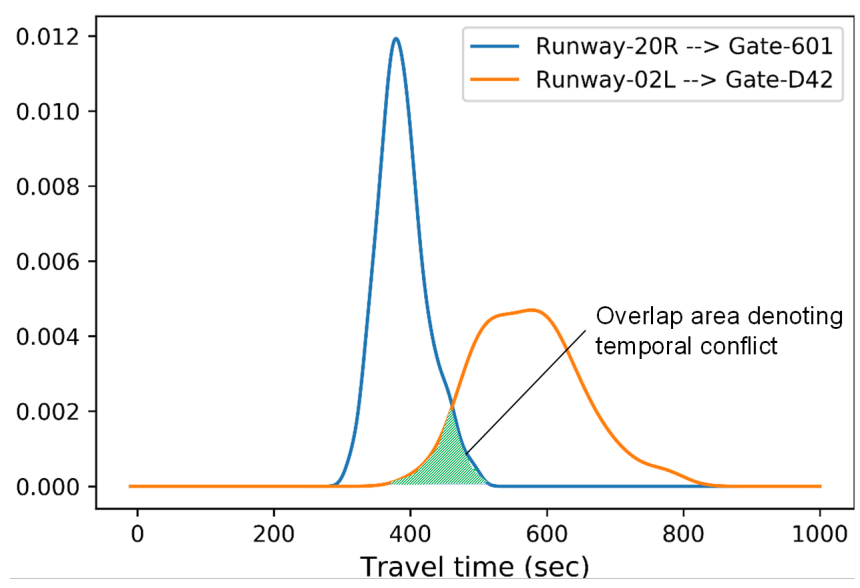

Fig. 9. Overlap area between 2 KDEs shown by hatched region: blue curve denotes travel time distribution (KDE) from runway 20R to intersection 5 (while going towards gate 601) and orange curve denotes travel time distribution (KDE) from runway $02 \mathrm{~L}$ to intersection 5 (while going towards gate D42).

In other words, $C_{t, i}$ is the common area between Gaussian kernel density curves corresponding to flights' travel time to a particular intersection. For instance, in Fig. 9, overlap area common between the curves at intersection 5 is 0.11 . In other words, if both the arriving aircraft start towards their respective destination gates at the same time, then there is an 11 per cent chance of them temporally conflicting at intersection 5 . 


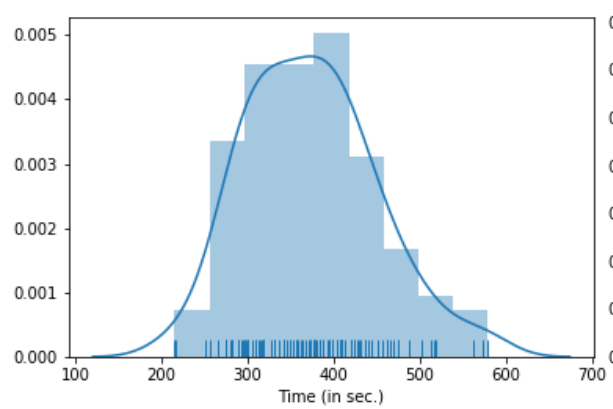

(a) Runway-20R \& Gate-601

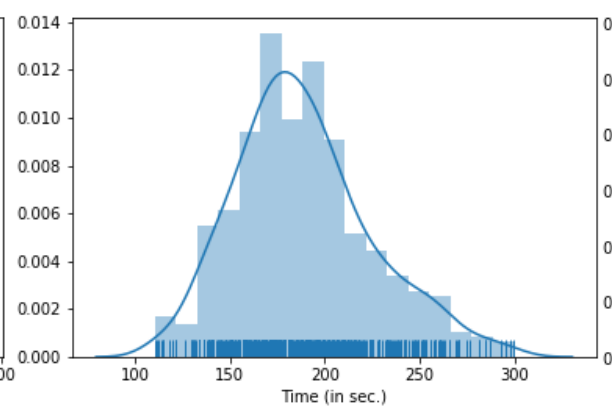

(b) Runway-02L \& Gate-601

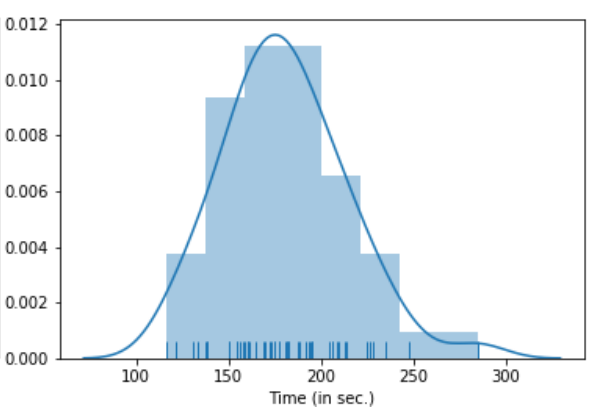

(c) Runway-02L \& Gate-401

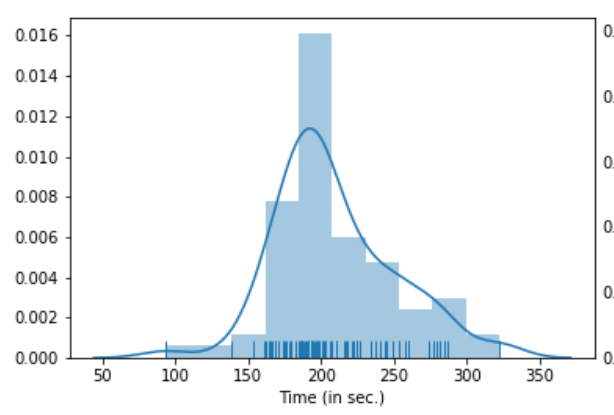

(d) Runway-02L \& Gate-C30

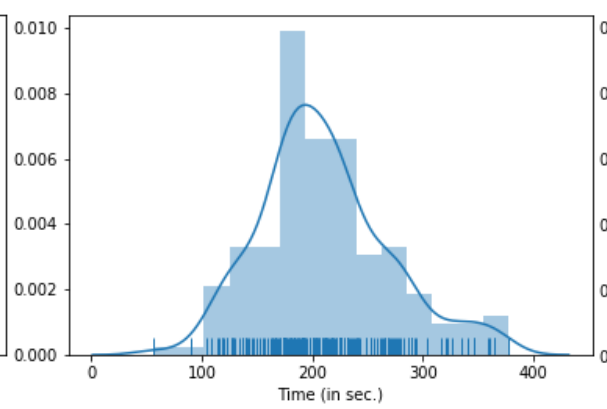

(e) Runway-02C \& Gate-C36

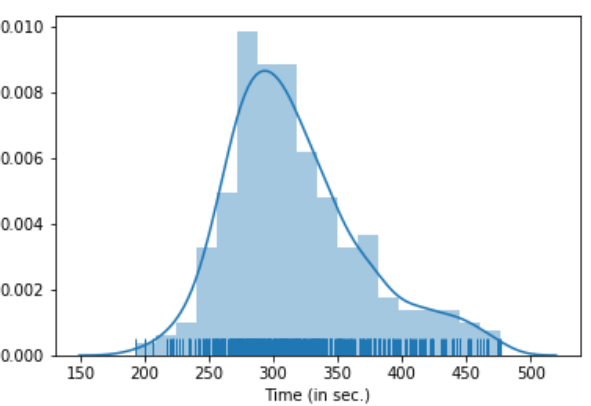

(f) Runway-20R \& Gate-D40

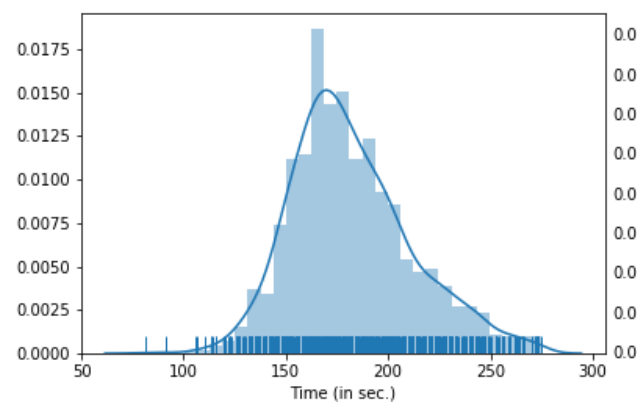

(g) Runway-02L \& Gate-D42

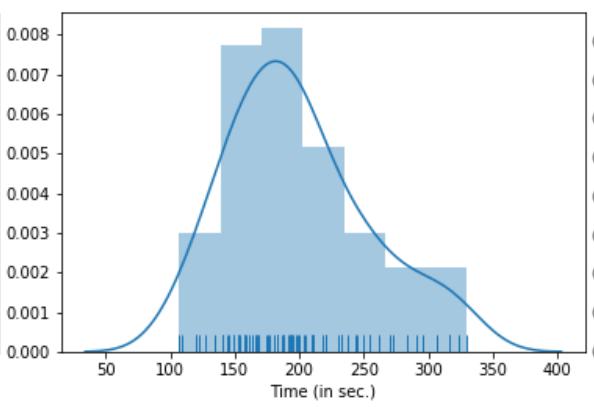

(h) Runway-02C \& Gate-C20

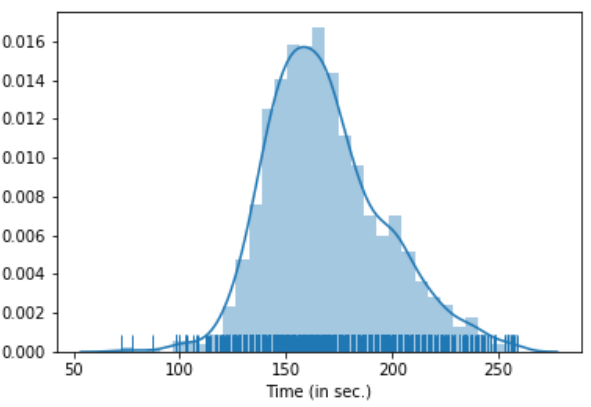

(i) Runway-02L \& Gate-E7

Fig. 8. Distribution of travel time of an arrival flight to intersection 5, for different origin and destination pairs. The blue curves are Gaussian Kernel Density Estimates fitted to observed travel time frequencies (to intersection 5) depicted by blue histograms.

3) Hot Spot score: Hot spot score, $H_{i}$ is a measure of potential conflicts (both spatial and temporal) associated with intersection $i$. The hotter the intersection depicted, the higher is its conflict potential. It is computed for all flights $f$ active in the sliding time frame (refer IV-L).

$$
\begin{array}{r}
H_{i}=C_{s, i} * C_{t, i} \\
\forall i \in I
\end{array}
$$

By equations 3, 4 and 5

$$
\begin{array}{r}
H_{i}=\sum_{a \in f} \sum_{b \in f}\left[P_{i}(\alpha) * P_{i}(\beta)\right] * \varphi_{i}(a, b) \\
\forall i \in I, a \neq b
\end{array}
$$

Where,

- $I$ represents total number of intersections.

The above model is run for a day's flight schedule which consists of information about origin, destination and start time of different flights. This gives probability of conflicts at intersections whose conflict potential or hotness increases when multiple flights are expected to cross the intersection at the same time.

\section{Results AND OUtLOOK}

As the time (window) progresses through the day, hot spots turn active and dormant. The more active intersections are shown as brighter red regions, while the dormant ones are depicted in pale yellow color (refer Fig. 10 and Fig. 11). In Fig. 10, hot spots predicted for ground traffic between 5 AM to 9:30 AM, are depicted on the airport diagram in 30 minute intervals. The color bar on the right of every sub-plot depicts numerical hotness values corresponding to the color shown on the aerodrome diagram on a scale of 0 to 7 . It can be observed that hot spots gradually become active and at 7:30 AM the hotness intensity piques up across the taxiway network. The hotness then gradually dies down. 


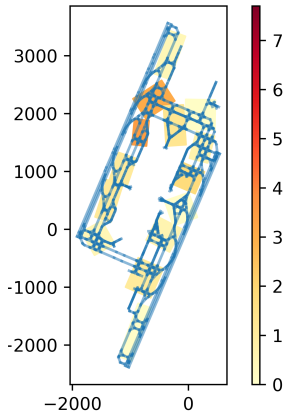

(a) 5:00 AM

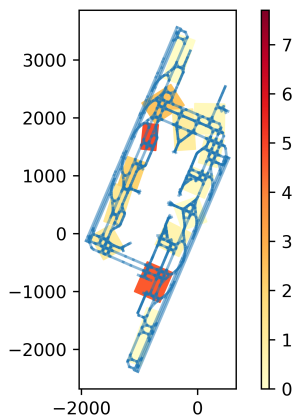

(b) 5:30 AM

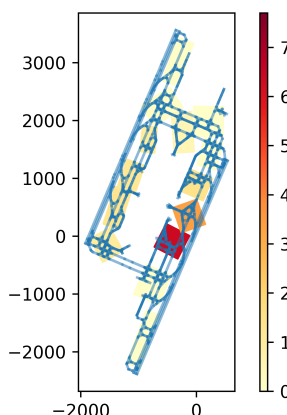

(c) 6:00 AM

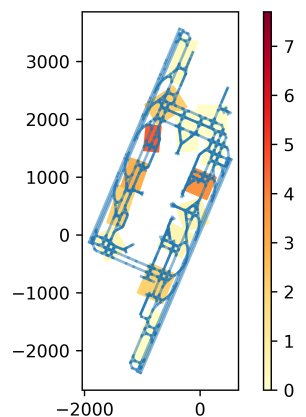

(d) 6:30 AM

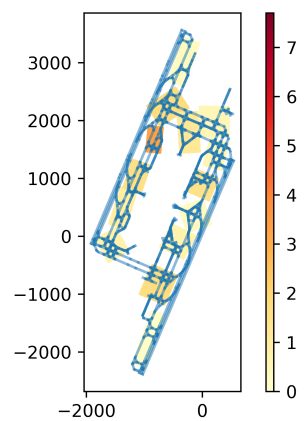

(e) 7:00 AM

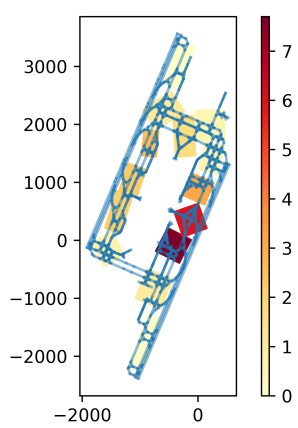

(f) $7: 30 \mathrm{AM}$

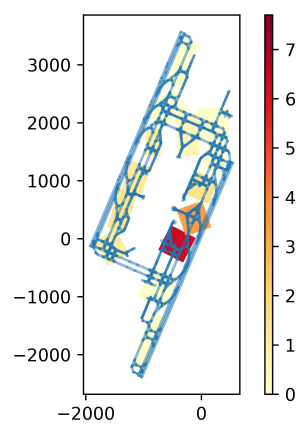

(g) 8:00 AM

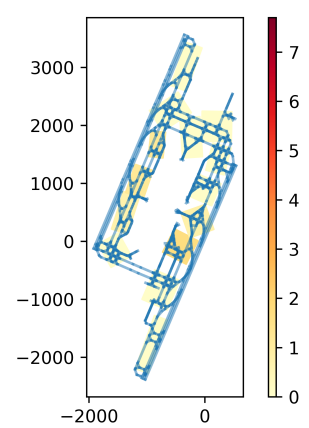

(h) $8: 30$ AM

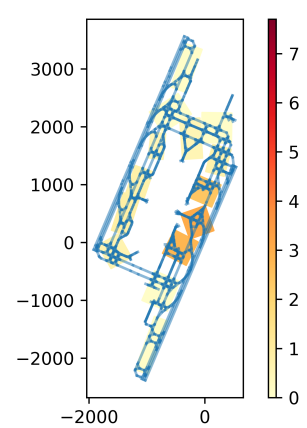

(i) 9:00 AM

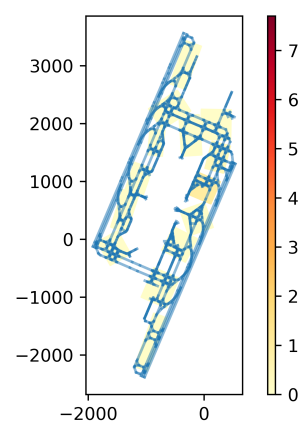

(j) 9:30 AM

Fig. 10. Hot spot chart for Changi airport ground traffic from 05:00 AM to 09:30 AM on 15-Dec-2017

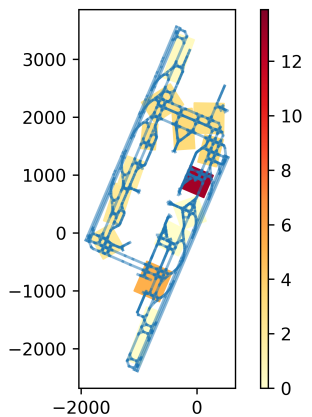

(a) 10:00 AM

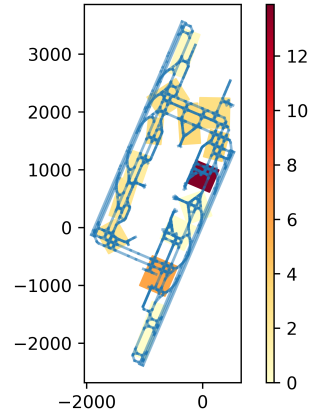

(b) 10:30 AM

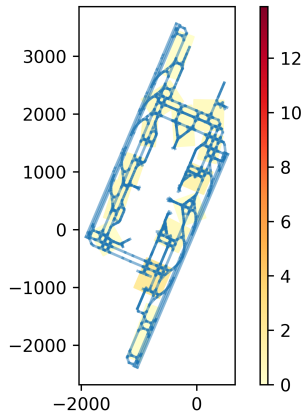

(c) 11:00 AM

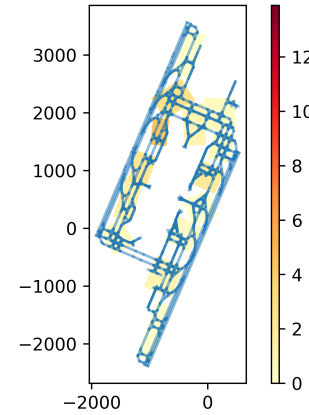

(d) 11:30 AM

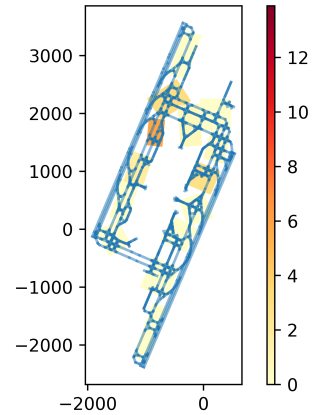

(e) 12:00 PM

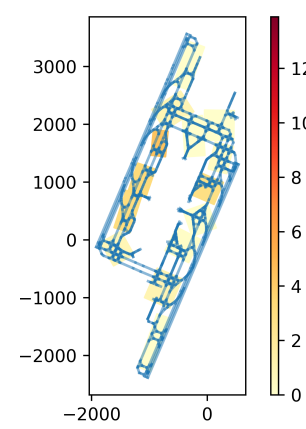

(f) $12: 30 \mathrm{PM}$

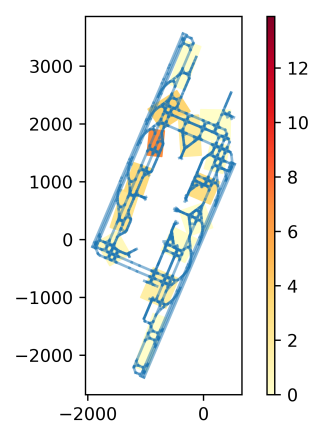

(g) 1:00 PM

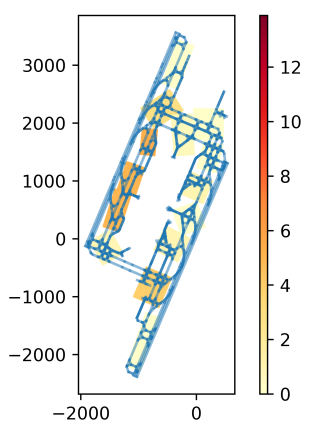

(h) 1:30 PM

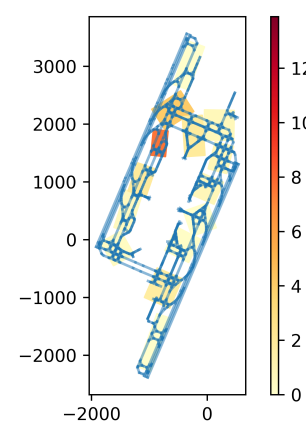

(i) 2:00 PM

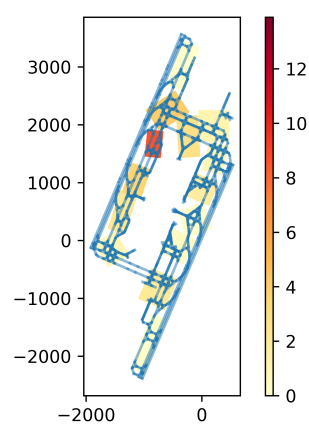

(j) 2:30 PM

Fig. 11. Hot spot chart for Changi airport ground traffic from 10:00 AM to 02:30 PM on 15-Dec-2017

In Fig. 11, hot spots predicted for ground traffic between $10 \mathrm{AM}$ to 2:30 PM, are depicted on the airport diagram in 30 minute intervals. The color bar on the right of every sub-plot depicts numerical hotness values corresponding to the color shown on the aerodrome diagram on a scale of 0 to 14. It can be observed that hot spots are very active at $10 \mathrm{AM}$ and 10:30 AM. The relative hotness then gradually dies down.

It can be clearly observed that hot spots change dynamically throughout the day. Hot spots, with lower hotness values, are observed in the early morning hours, (even) when Changi 
airport witnesses lots of traffic. This shows that it is certainly possible to safely manage huge traffic flows. Around $10 \mathrm{AM}$, hotness intensity again piques up. It is worth noting that hot spots around runway-taxiway intersections are generally brighter and often more active than those father away from the runways. This can be attributed to the fact that taxiway intersections closer to runway exits (and entry points) serve greater number of aircraft. As these areas are busier, they turn hot more often.

These results, which are based on travel time approximation by Gaussian distributions, were shown to a Ground Movement Air Traffic Controller. In his subjective opinion, highlighted hot spots make sense and leads to better understanding of taxiway movements and increased situational awareness. However, we plan to perform an in-depth human-in-the-loop validation of the dynamic hot spot model by ATCOs in 360 degree tower simulator. Future research shall incorporate ground vehicle movements in the analysis as well to make the model more nuanced and realistic. It will be interesting to see emergent hot spot patterns in Changi airport.

\section{REFERENCES}

[1] ICAO. Safety Reports. 2019, https://www.icao.int/safety/Pages/SafetyReport.aspx.

[2] IATA. IATA Forecast Predicts 8.2 billion Air Travelers in 2037. 2018, https://www.iata.org/pressroom/pr/Pages/2018-10-24-02.aspx.

[3] Steffen Kaspers, Nektarios Karanikas, Alfred Roelen, Selma Piric, and Robert J De Boer. How does aviation industry measure safety performance? current practice and limitations. International Journal of Aviation Management, 4(3):224-245, 2019.

[4] Saqib Gulzar, Hasnain Ali, and Chetan Doddamani. A conceptual framework for introducing 'mobility as a service' in india: opportunities challenges. In Proceedings of ASCE India Conference 2017. Indian Institute of Technology Delhi, 2017.

[5] Saqib Gulzar, Hasnain Ali, and Chetan Doddamani. Ant colony optimization in pavement asset management. In Proceedings of ASCE India Conference 2017. Indian Institute of Technology Delhi, 2017.

[6] Michael Schultz, Judith Rosenow, and Olive Xavier. A-cdm lite: situation awareness and decision making for small airports based on ads-b data. [in press].

[7] ICAO Doc. 4444 pans-atm fifteenth edition 2007, chapter 4. Paragraph, 4(1), 2007.

[8] Flight Safety Foundation. Runway safety hot spots list, 2010.

[9] J Schönefeld and DPF Möller. Runway incursion prevention systems: A review of runway incursion avoidance and alerting system approaches. Progress in Aerospace Sciences, 51:31-49, 2012.

[10] Mary E Johnson, Xun Zhao, Brian Faulkner, and John P Young. Statistical models of runway incursions based on runway intersections and taxiways. Journal of Aviation Technology and Engineering, 5(2):3, 2016.

[11] Sybert H Stroeve, Pradip Som, Bas A van Doorn, and GJ Bert Bakker. Strengthening air traffic safety management by moving from outcomebased towards risk-based evaluation of runway incursions. Reliability Engineering \& System Safety, 147:93-108, 2016.

[12] Steven D Young and Denise R Jones. Runway incursion prevention: A technology solution. 2001.

[13] GK Singh and Christoph Meier. Preventing runway incursions and conflicts. Aerospace science and technology, 8(7):653-670, 2004.

[14] Peter N Squire, Jane H Barrow, Kevin T Durkee, Carl "Mac" Smith, Jennifer C Moore, and Raja Parasuraman. Rimdas: A proposed system for reducing runway incursions. Ergonomics in Design, 18(2):10-17, 2010.

[15] Denise R Jones, Cuong C Quach, and Steven D Young. Runway incursion prevention system-demonstration and testing at the dallas/fort worth international airport. In 20th DASC. 20th Digital Avionics Systems Conference (Cat. No. 01CH37219), volume 1, pages 2D2-1. IEEE, 2001.
[16] Harshad Khadilkar and Hamsa Balakrishnan. Network congestion control of airport surface operations. Journal of Guidance, Control, and Dynamics, 37(3):933-940, 2014.

[17] Andrew T Ford and Timothy P Waldron. Relating airport surface collision potential to taxiway geometry and traffic flow. In 14th AIAA Aviation Technology, Integration, and Operations Conference, page 2156, 2014.

[18] Timothy P Waldron, Andrew T Ford, and Sherry Borener. Quantifying collision potential in airport surface movement. In 2013 Integrated Communications, Navigation and Surveillance Conference (ICNS), pages 112. IEEE, 2013.

[19] Zhenghong Xia, Bo Zheng, Jian Wan, and Xinping Zhu. Recognition algorithm and risk assessment of airport hotspots. Journal of Shanghai Jiaotong University (Science), pages 1-6, 2019.

[20] Hasnain Ali, Yash Guleria, Sameer Alam, and Michael Schultz. A passenger-centric model for reducing missed connections at low cost airports with gates reassignment. IEEE Access, 7:179429-179444, 2019.

[21] Hasnain Ali, Yash Guleria, Sameer Alam, Vu N Duong, and Michael Schultz. Impact of stochastic delays, turnaround time and connection time on missed connections at low cost airports. In Proc. 13th USA/Eur. Air Traffic Manage. R\&D Seminar, 2004.

[22] Hasnain Ali and Arpan Kumar Kar. Discriminant analysis using ant colony optimization-an intra-algorithm exploration. Procedia computer science, 132:880-889, 2018.

[23] K Chidananda Gowda and G Krishna. Agglomerative clustering using the concept of mutual nearest neighbourhood. Pattern recognition, 10(2):105-112, 1978.

[24] David M Bashtannyk and Rob J Hyndman. Bandwidth selection for kernel conditional density estimation. Computational Statistics \& Data Analysis, 36(3):279-298, 2001.

[25] Bernard W Silverman. Density estimation for statistics and data analysis. Routledge, 2018. 